\title{
Post-approval Change Submission: ICH Q12 Impact Analysis on India and Japan
}

\author{
S. MOORKOTH* AND T. HALDAR \\ Department of Pharmaceutical Quality Assurance, Manipal College of Pharmaceutical Sciences, Manipal University, \\ Manipal-576 104, India
}

Moorkoth and Haldar: Post-approval Change Submission

\begin{abstract}
The present research provides a discussion on post approval change management of pharmaceuticals, biologics and drug device combinations, which is an important part of life cycle management. The present study focuses on understanding the existing post approval change management system in India and Japan. Comparison is then made with the elements and principles provided in draft ICH Q12 guideline. In November 2017 the draft guidance ICH Q12 entitled "Technical and Regulatory Considerations for Pharmaceutical Product Lifecycle Management" has been published for comment. The discussion of this study would bring to light the key requirements, challenges and impact of future implementation of ICH Q12 in India and Japan. The initiative taken by ICH Q12 with this guideline focuses mainly on harmonizing the post approval system throughout the world which has the potential to reduce costs and time burdens for the regulators and industry.
\end{abstract}

Key words: Post-approval changes, ICH Q12, pharmaceutical industry, regulatory aspects, change management, harmonization, life cycle management

After receiving approval for drug commercialization from the concerned regulatory authority (RA) of a country, the manufacturer or market authorization holder (MAH) might realize and propose changes to the contents of a registered dossier. These changes can be administrative change or chemistry manufacturing and controls (CMC) changes, which need prior approval from the concerned RA if the proposed change is considered to have an impact on the safety, efficacy and quality of the drug. These changes are informed to the concerned authority for scrutiny of the changes proposed by filing application referred to as post approval change submissions ${ }^{[1]}$.

Present scenario of the post market change management system as studied shows that different jurisdictions have different mechanisms on change management systems as well as different approaches for reporting the proposed changes to concerned health authority. Each country having different mechanisms pose a challenge and immense burden on manufacturers to maintain a steady supply of same products in different the countries. Change is an inevitable part in the life cycle management of a product which can occur due to causes such as revisions of regulatory guidelines,

*Address for correspondence

E-mail: moorkoth.s@manipal.edu

January-February 2020 changes in manufacturing process due to innovation of efficient and cost reduction methods, changes in business and product models, changes in analytical and formulation specifications and many other reasons. All these changes revolve around improving the safety and quality of healthcare products delivered to the consumers.

Many countries have their own regional regulations for post market change submissions. In the United States of America the 'Scale up and post approval changes' guidance provides information on various aspects of post approval changes. This guidance provides recommendations on new drug applications or abbreviated new drug applications on the levels of post approval changes, recommended tests and documentations for CMC changes. The implementation of this guideline has led to faster approval times and incorporation of the post approval changes of already

This is an open access article distributed under the terms of the Creative Commons Attribution-NonCommercial-ShareAlike 3.0 License, which allows others to remix, tweak, and build upon the work non-commercially, as long as the author is credited and the new creations are licensed under the identical terms

Accepted 03 November 2019

Revised 29 June 2019

Received 19 April 2019

Indian J Pharm Sci 2020;82(1):2-10 
marketed product ${ }^{[2,3]}$. The changes are categorized based on risk as level 1, 2 and 3 for major, moderate and minor changes, respectively.

The European Medicines Agency guidelines have given rules for post approval changes known as variation filing in Europe and have classified the variations. Type 1A variations are minor variations that are submitted annually and does not require immediate notification to the competent authority. Whereas the type $1 \mathrm{~A}$ IN variations need to be notified immediately. The type IB variations are moderate level changes that must be notified to the competent authority. Type II or major variations are high risk changes for which prior approval from competent authority must be taken before implementation of the change $\mathrm{e}^{[4]}$.

India and Japan have different regulatory systems for management of post approval system. In India, post approval changes for new chemical entities are not classified based on risk and impact of change on the safety and efficacy of pharmaceutical product ${ }^{[5,6]}$. Post approval changes are submitted to an online platform called the Sugam Portal, which provides a template list of changes and checklist of required documents to be submitted ${ }^{[7]}$. Post approval changes for biologics are classified based on risk, into level I supplements, level II notifiable changes and level III annual notification ${ }^{[5]}$. Whereas, in Japan post approval changes are submitted as an application for making changes in a registered master file (MF) of a marketed drug product. Change applications are classified into partial change application and minor change application ${ }^{[6,8]}$.

The International Council for Harmonization of Technical Requirements for Pharmaceuticals for Human Use (ICH) is an organization that brings together all the regulatory authorities of countries around the world and pharmaceutical industry to discuss about various developments and innovations regarding technical and scientific characteristics of drug product development and registration ${ }^{[9]}$.

In 1989, the three founding member countries Europe, Japan, and the United States started discussing on harmonization of regulations and standards for pharmaceuticals. ICH was created in April 1990 at a meeting in Brussels. Initially the reason was to coordinate the regulatory activities of the European, Japanese and United States regulatory bodies in consultation with the pharmaceutical industries of these regions, to talk over and agree over the scientific aspects arising from product registration. It has then developed over years and has added many countries throughout the world and conduct worldwide meetings to discuss on bringing development, promote innovation and harmonization of the technical regulations governing the safety and efficacy of health care products like drugs and biologics ${ }^{[9]}$. This is achieved by developing guidelines which are written after intense scrutiny and discussion by industry and regulatory experts worldwide. Besides the three founding members there are other industry regulatory members and observers.

The formal harmonization process for the initiation, drafting and completion of an ICH guideline has a proper procedure in place ${ }^{[10]}$. In step 1 , the expert working group of a particular topic prepares a consensus draft of the technical document. The draft is then forwarded to the steering committee. Step 2 is reached as soon as the consensus is confirmed and all the regulatory members take required actions to draft the guideline. In step 3 the draft guideline is then put up for regulatory consultation by the ICH regions. Discussions are then conducted on comments provided by all the experts. The changes are incorporated in the draft guideline and then the experts draft guideline is finalized. In step 4, the finalized experts draft guideline prepared by the expert working group is then submitted to the steering committee of the ICH assembly for the adoption of the $\mathrm{ICH}$ harmonized guideline. After the adoption of the harmonized guideline regulatory implementation of the guideline is carried out in step 5 .

There are many guidelines that have been harmonized on 4 main topics like safety (S-Guideline), quality (Q-Guideline), efficacy (E-Guideline) and multidisciplinary (M-Guideline). Despite the harmonization of many guidelines and their implementation in the various member countries, the members also have their own local regulations and guidelines on a variety of topics on which harmonization effort is still under process.

Pharmaceutical life cycle management is the strategy for management of a specific pharmaceutical product from development to final withdrawal from the market. It consists of a succession of stages and implementing strategies at each stage for maintaining the steady supply of the product in market throughout the life cycle. During the life cycle of a medicinal product there may be changes due to quality improvement, changes in manufacturing methods, cost changes, process optimization or changes due to innovation of technology. When a change is made to a pharmaceutical product it is necessary to ensure that the product must meet the relevant regulatory requirements by submitting 
changes for review to the regulatory agency and remain compliant ${ }^{[11]}$.

The different phases of pharmaceutical product life cycle are, pharmaceutical industry development, pharmaceutical product development, manufacture of the product, compilation of the common technical document (CTD or eCTD), submission of the dossier to concerned RA, marketing approval of the product by RA, post approval compliance, post approval changes on marketed products (if any) and renewals of product marketing licenses (after expiry of license) ${ }^{[12]}$.

Throughout the lifecycle of a pharmaceutical product, the license holder is responsible for the product circulating in the market place and is also required to make technical and scientific advances. The license holders may wish to alter/improve the pharmaceutical product also called as post approval changes, which must be implemented after regulatory scrutiny.

On 16th November 2017, the draft guideline ICH Q12 entitled "Technical and Regulatory Considerations for Pharmaceutical Product Lifecycle Management (PLCM)" has been published for comment ${ }^{[13]}$. The scope of this guideline applies to pharmaceutical drug substances, drug products, biological products and drugdevice combination products. This guidance proposes a harmonized approach for lifecycle management that will benefit industries, regulatory authorities and patients by providing an outline to enable the management of post approval changes enabling a foreseeable and wellorganized approach. The guideline provides new tools to streamline and harmonize processes for post approval changes. The guideline also talks about implementing these tools along with supporting ICH guidelines Q8, Q9, Q10, and Q11 to develop a more enhanced quality by design (QbD) approach for post approval submissions. The QbD concept is that the quality cannot be tested into the product, but it should be built into $\mathrm{it}^{[14]}$. This can be adopted so that the post approval changes that are within a defined limit can be implemented with regulatory flexibility. This will benefit the industry by decreasing the number of supplements required for making changes to already marketed products. This will allow the continuous implementation of changes without delay ${ }^{[14]}$.

The study discusses about the current regional change management regulations in India and Japan and how the future implementation of ICH Q12 might impact the regulatory systems in both these countries. The challenges that are currently faced to implement ICH Q12 in these 2 countries are the main focus of the study.
Since Japan is one of the founder members of $\mathrm{ICH}$ and India being the observer ${ }^{[15]}$, comparison between existing regulations of these two countries with the ICH Q12 requirements were made to discuss how the level of involvement with ICH has benefited each country in bringing about harmonization of post approval change management systems.

The ICH Q12 guidance document was collected from $\mathrm{ICH}$ website. The requirements mentioned in the guideline was reviewed and understood. ICH Q12 annex contains illustrative examples for easy interpretation of the requirements mentioned in the guideline. The regulatory guidance documents for post approval change submissions provided by health authorities of India and Japan were also reviewed and understood. The requirements given in the Central Drugs Standard Control Organization (CDSCO) guidance for post approval change submissions in India was compared with that of requirements in ICH Q12 guideline. Online submission portal, the Sugam portal was accessed to understand the requirements and submission process for post approval changes. For Japan, the Pharmaceutical and Medical Devices Agency (PMDA) guidelines were compared with that of ICH Q12 requirements. Various websites and industry presentations based on this topic was referred to get a deep understanding of the current challenges for future implementation of ICH Q12 in Japan and India.

The draft guidance on ICHQ12 has provided harmonized regulatory tools and enablers which will enhance industry's ability to manage post approval changes in a more predictable and efficient manner ${ }^{[13]}$. Following the ICH Q12, most manufacturing and analytical changes can be managed efficiently under the pharmaceutical quality system (PQS) of a company without regulatory approval prior to implementation. The following tools are expected to be utilized by manufacturers all over the world for the management of post approval changes upon implementation of ICH Q12 ${ }^{[13]}$.

A well characterized risk based classification of changes having impact on drug safety and efficacy is crucial for appropriate regulatory communication for the proposed changes. This categorization provides a clear understanding of requirements and is important to the effective use of industry and regulatory requirements. The changes must be categorized into prior approval (changes with high risk which requires regulatory review prior to implementation) and notification changes (moderate to low risk changes not requiring regulatory review prior implementation). In addition to 
this lowest risk changes can managed within the PQS of a company and not reported to regulators, but may be verified upon routine inspection by authority ${ }^{[13]}$.

Established conditions (EC) are the elements in a CTD of a product, which are considered necessary to assure product quality. Any change to EC's requires a submission to a RA. ECs are defined along with their reporting categories, justification for selection of reporting category and supportive information in the relevant sections of the CTD in module 3. ECs in a submission can be either implicit (derived from regional regulations) or explicit (defined by the manufacturer). The original manufacturing authorization application (MAA) submission incorporated with EC's and supportive information are reviewed and must be approved by the authority ${ }^{[13]}$.

Post approval change management protocol (PACMP) is one of the useful tools that offers predictableness concerning the evidence essential to support an anticipated CMC change along with the associated reporting category, on previous arrangement between the manufacturing authorization holder and RA. A protocol describing how an anticipated CMC change can be organized and confirmed, including the assessment of impact, specifications, acceptance criteria and the suggested reporting category should be submitted by the manufacturer to the RA along with original manufacturing authorization application or as a supplement ${ }^{[13]}$. Different steps of PACMP are given in fig. 1. A PACMP may include single or multiple changes for a single product, or can also include multiple changes to be applied to multiple products. The modification to an approved PACMP can be submitted as a protocol modification or submission of a new protocol as decided by RA.

$\mathrm{PLCM}^{[13]}$ document outlines the plan for product life cycle management proposed by MAH. The document includes the summary of product control strategy, ECs and their proposed reporting categories, PACMP (if used) and other post approval CMC commitments. This document is updated throughout the life cycle of the product as needed. The PLCM document can be located anywhere in the CTD Module 1, 2, or 3 based on regional regulations.

CDSCO under the Ministry of Health and Family welfare of the Indian Government oversees the drug regulations and marketing requirements in India. The general goal is to ensure that the medicinal products are of satisfactory quality, safety and efficacy and are manufactured and distributed in such a way so that the attributes does not change until it reaches the patient or consumers $^{[5,7]}$.

With the implementation of Sugam Portal on 14th November 2018, intended for filing applications for

\section{Application of PACMP}

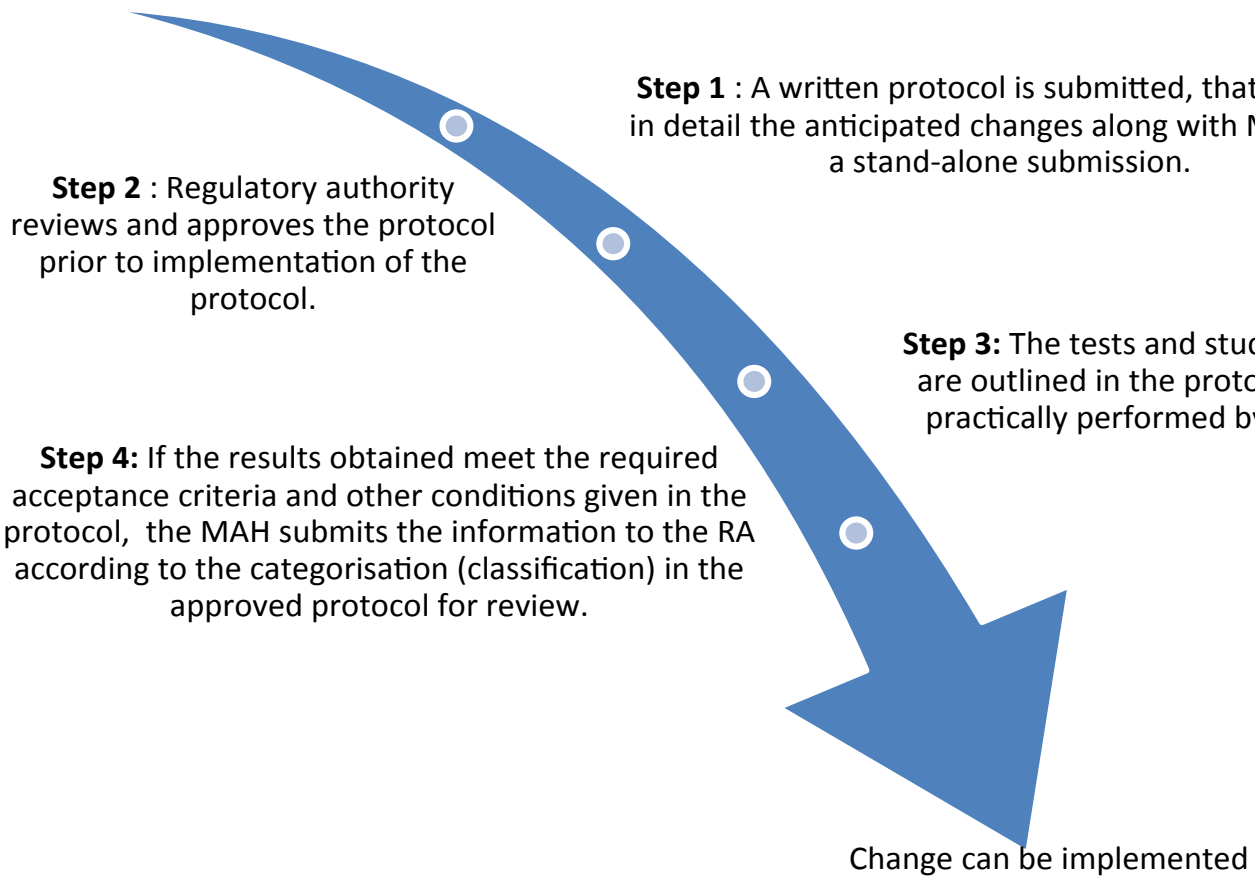

Fig. 1: Process flow of post approval change management protocol (PACMP) procedure ${ }^{[13]}$ 
all the services rendered by CDSCO the post approval changes are now submitted online through the portal for both drugs and biologics ${ }^{[5,7]}$. The portal shows a list of changes that can be implemented along with templates and document checklist to be uploaded for the review and approval of the requested change. For assistance on changes that are not included in the list, the sponsors are advised to write a query to the Drugs Controller General of India.

Post approval change system in India is not well categorized based on risk based and scientific based approach for new chemical entities. Whereas, for biologics post approval changes are well classified as given in Table $1^{[5]}$.

CDSCO is an observer in $\mathrm{ICH}^{[15]}$. The observers have right to attend meeting but have no voting rights during the meeting decisions. An observer can delegate a maximum number of two persons to attend the assembly meetings. The observers also have the right to appoint experts for working in the ICH working groups ${ }^{[16]}$.

In India the post approval change submission system is not categorized as per science based and risk based classification for new chemical entities, whereas there is a classification system available for biologics. The absence of a formal risk based classification of changes that impact the safety and efficacy of the product for new chemical entities is one of the major drawback as it creates confusion for industries as to which changes need to be reported prior to implementation. Classification of changes based on risk will help regulatory authorities to prioritize those tasks that will need an extensive review before these high risk changes can be implemented to the commercial products. Upon implementation of ICH Q12 in India, there is a possibility for categorization of the changes based on risk for NCEs. This will streamline the procedure for submitting changes and help agency for faster review and approval of changes without hindering the steady supply of the medical products.

Current post approval change submission system in Sugam portal, has a list of changes that can be implemented by submitting their respective checklists of documents online. The changes that are not listed online are submitted offline through hard copies to CDSCO or zonal state food and drug administrations. Some of the changes that are listed usually do not require extensive review times as these do not affect the safety and efficacy of the drug. Upon implementation of ICH Q12 and risk based categorization of the changes, it is expected that there might be changes on the portal with different sections addressing different changes based on the risk classification and each section having a different review timeline. This will ease up and fasten the process of post approval change submission. But since the Sugam portal is newly established, making changes to it might take up lot of resources and time due to website development and formal procedures of the government.

Regulatory system and post approval life cycle system in India are not stringent and well managed. Implementation of ICH Q12 and the proposed tools in India requires proper planning, establishment and resources for implementation of these tools. There is a concern that the proposed tools in ICH Q12 guideline is not completely attuned to the already established legal framework/national regulations in India with regard to the usage of EC in CTD, PACMP and PLCM documents. In order to fully realize the impact of ICH Q12 regional regulations in India will need to take strides in building an appropriate infrastructure and update existing guidelines to align with the tools and enablers mentioned in ICH Q12 guideline. Moreover, it is not clear how the MAH will have to establish the EC, the PLCM document and PACMPs and have these reviewed, negotiated and approved by CDSCO. Specifically, as these mechanisms are not discussed in current CDSCO guidelines.

Post approval regulatory actions are complex, resource intensive and expensive. Implementing this will require qualified people in various areas, resources to maintain the system functions efficiently for both regulators and manufacturers.

\section{TABLE 1: CLASSIFICATION OF POST APPROVAL CHANGES FOR BIOLOGICS IN INDIA ${ }^{[5]}$}

\begin{tabular}{ccc}
\hline S.no & Type of change & Impact on purity, safety efficacy and potency of biologics \\
1. & Level 1 & $\begin{array}{c}\text { High impact. Requires extensive documentation and their assessment by the DCGI before the } \\
\text { implementation of these changes to the marketed products. These documentation is also called } \\
\text { as "Supplements" }\end{array}$ \\
2. Level II & $\begin{array}{c}\text { Medium impact. Recommended supporting documents should be filed along with the changes to } \\
\text { the DCGI. These are also called as "Notifiable changes" }\end{array}$ \\
3. & $\begin{array}{c}\text { Minimal impact. These changes can be incorporated without the prior review by the regulatory } \\
\text { authority, DCGI. The supporting data is submitted annually, however upon request by DCGI } \\
\text { should be provided within fifteen days }\end{array}$ \\
\hline
\end{tabular}


There might be ambiguity for the manufacturer during categorization of some new or innovative changes and may require consulting from the RA. Thus a post approval change consultation system must be updated or introduced by the CDSCO so that the manufacturer can get a clear idea on how to proceed with the implementation of the change.

The PMDA, a subsidiary body under the Ministry of Health Labour and Welfare (MHLW) is responsible for the pharmaceutical regulatory affairs in Japan ${ }^{[8]}$. Among many other things the agency is tasked with clinical studies, scientific review of market authorization applications and their approvals, licensing, post approval changes and post market safety surveillance. The Drugs and Medical Devices Law is the main law concerning to the pharmaceutical products and medical devices in $\operatorname{Japan}^{[8]}$.

The MF system is used by foreign manufacturers or manufacturers in Japan for drug substances to separately submit the information to register the data regarding the quality, efficacy and manufacturing of the drug substance for review by the health authority. The registered data is cited as the essential data for an approval review of the pharmaceutical product in which the drug substance is used. MF system protects the intellectual property during license application and review. Items like drug substances, preservatives, additives, raw materials, processing materials can be registered through this system. Foreign manufacturers or manufacturers in Japan can register in MF by submitting the specified forms called application form for MF registration ${ }^{[17]}$.

An overseas drug substance manufacturer or foreign manufacturers of drug substances is allowed to apply for registration in MF system. The foreign/overseas manufacturer has to obtain a foreign manufacturer accreditation. The accreditation number, category and the date of accreditation of the foreign manufacturing facility must be provided in the MF registration application form.

A foreign manufacturer must also appoint a person or company that can accept the responsibilities regarding the relevant MF registration with an address within Japan. This person is known as in-country caretaker of drug substances. The person/company that is responsible for providing the data in the MF is called the MF holder. The person who is responsible for only the manufacturing or marketing of the drug is known as the MAH. The MF holder and MAH can be the same person or different person depending on case by case basis.

The Japanese MF has two distinct parts, an open (disclosed part) in which the information presented in this part of the MF can be disclosed to the MAH, if they are different person. And closed (restricted part) in which the information presented in this part of the MF cannot be disclosed to the MAH, if they are a different person. The company that manufactures the active pharmaceutical ingredient can only be the MF holders. Manufacturers or contract manufacturers that perform repackaging, packing, labelling, storage, examination cannot be the MF holders ${ }^{[17]}$.

Along with the application to change of the MF that is submitted, the manufacturing/marketing authorization holder must also submit a partial change application or a slight modification notification for the MF depending on the contents of the change. In either case, the MF registrant must notify the manufacturing/marketing authorization holder of the change(s) in advance through the MF in-country caretaker (in case of foreign manufacturer). In case the changes in the items recorded in MF has a potential to significantly change the nature of drug substances then it is mandatory that a new MF registration form must be submitted and not a change of registration. The three levels of changes classified are given in Table $2^{[17]}$.

The PMDA under MHLW is one of the founding regulatory members of the ICH. The Japan Pharmaceutical Manufacturers Association (JPMA) established in 1968 represents the research and development based pharmaceutical industries in $\mathrm{Japan}^{[18]}$. JPMA is one of the founding industrial members of the $\mathrm{ICH}^{[15]}$. JPMA plays very important roles at ICH providing and aiming for the international harmonization of pharmaceutical regulations. The founding members have the right to vote for important decisions during the assembly meetings, appoint members in all the ICH working groups and support the visions and aims of the organization ${ }^{[16]}$.

The post approval change system in Japan is well categorized based on risks and impact of change in the quality of the drug product. They are categorized as partial changes that are major changes which require regulatory scrutiny before implementation and minor change notification for minor changes that have low impact on drug quality. Other low level changes are recorded in the standard operating procedures in the pharmaceutical quality management system and are reviewed during annual audits. Thus regulations in 
TABLE 2: CLASSIFICATION OF POST APPROVAL CHANGES OF DRUG SUBSTANCES IN JAPAN ${ }^{[17]}$

\begin{tabular}{|c|c|c|c|c|}
\hline S. no & Type of change & $\begin{array}{c}\text { Impact on quality safety and } \\
\text { efficacy }\end{array}$ & Reporting timeline & Documents submitted \\
\hline 1. & $\begin{array}{l}\text { Significant } \\
\text { changes in nature } \\
\text { of drug substances }\end{array}$ & Significant & - & New MF Application \\
\hline 2. & Partial changes & High & $\begin{array}{l}\text { Prior approval from PMDA required } \\
\text { before implementing changes }\end{array}$ & $\begin{array}{l}\text { Partial change } \\
\text { application }\end{array}$ \\
\hline 3. & Minor changes & Low & $\begin{array}{c}\text { Within } 30 \text { days after implementing } \\
\text { changes to PMDA }\end{array}$ & $\begin{array}{l}\text { Minor change } \\
\text { notification }\end{array}$ \\
\hline
\end{tabular}

Japan meets the most important requirement of ICH Q12 tool, that is risk based categorization of changes and thus already meets the criteria described in Q12 guideline.

Most of the variations are partial change applications which lead to long review approval times and also expenditure of large amount of resources. This causes delays in change approvals of marketed products which might interfere with the steady flow of the drugs in the country.

In the Japanese New Drug Application there is a difference in Module 1 compared to Module 1 of other ICH regions. It contains a summary of most important elements of the application. The content that is provided in the Module 1.2 of the CTD is called as matters subject to approval or Application Form (J-AF), which is a legally binding approved matter. Any change to the contents described in this section will require a filing of a change application as these changes are to be reviewed by the authority. Content of this part of the module includes all the critical and non-critical parameters of the manufacturing procedures of the drug product. The parameters that have a high significance of the target or set value on the quality of drug substance and product are enclosed in major $(<<>>)$ brackets ${ }^{[19]}$. In case the matter present between these brackets has to be changed, partial change application has to be filed. Those parameters that have low significance on the quality of drugs are enclosed in minor (" " or \lceil\rfloor$)$ brackets, change in the content enclosed in these brackets will result in minor change notification ${ }^{[19]}$. For other matters that are not enclosed in parenthesis, a partial change application has to be filed for implementing the change ${ }^{[19]}$. The ICH Q12 guideline explains a similar concept in the tool called EC, which mentions the importance of identifying important quality parameters and regulatory scrutiny on these parameters when they are subjected to changes. Thus streamlining the J-AF into format as described in EC in Q12 would be required which will include appropriate level of manufacturing process and specification sections. However reviewers in Japan expect a very detailed information in J-AF. This will be unnecessary if there are higher number of variations for low or no impact changes.

The application of PACMP as described in the Q12 guideline, provides an opportunity for faster and more predictable post approval change management system. In Japan there is no similar concept to accept a protocol on planned changes. It will be difficult to implement this concept without changing the current national regulations. The use of these protocols might also lead to downgrading of changes earlier classified as partial changes to minor change notifications.

The implementation of PLCM documents as discussed in the Q12 guideline should be considered. The location of PLCM document is not defined in current regional regulations in Japan. Maintenance and updating of the PLCM document may potentially add unnecessary duplication of work, regulatory burden and extensive resources. It is also not sure how the RA will approve and negotiate the PLCM document.

Post approval regulatory actions are complex, resource intensive and expensive. PQS and change management system should be made effective in order to fully realize the implementation potential of Q12 guideline. Miscommunication between manufacturer and $\mathrm{MAH}$ leads to improper change management, which leads to discrepancies in the information in J-AF.

Under the current systems, management of post approval changes in a global scale is time consuming complex, expensive and unpredictable. With an ever evolving industry such as the pharmaceutical industry, there is a hope that advances will always be made, technology be improved but this process will also probably result in an ever changing set of marketing authorization applications and legal guidelines. That is the reason there is a keen interest in the development of the ICH Q12 guideline which is expected to provide necessary tools for planning and strategizing post approval changes during the early phases of product life cycle management. With the development of 
TABLE 3: SUMMARY OF ICH Q12 IMPLEMENTATION STATUS IN INDIA AND JAPAN

\begin{tabular}{cccc}
\hline S.no & ICH Q12 tools and enablers & Status in India & Status in Japan \\
1. & $\begin{array}{c}\text { Categorization of post approval } \\
\text { chemistry CMC changes }\end{array}$ & $\begin{array}{c}\text { Only present for biologics. No } \\
\text { guideline states categorization } \\
\text { of drugs }\end{array}$ & $\begin{array}{c}\text { Already implemented for both drugs and } \\
\text { biologics. Categorized into "partial changes" } \\
\text { and "minor change notification" }\end{array}$ \\
2. & $\begin{array}{c}\text { Established Conditions (EC) } \\
\text { Post Approval Change }\end{array}$ & Not implemented & $\begin{array}{c}\text { Already present in the Module 1.2 (J-AF) of the } \\
\text { CTD is called as "Matters subject to approval” } \\
\text { No similar concept in regional regulations. } \\
\text { Considering to implement soon }\end{array}$ \\
3. & $\begin{array}{c}\text { Management Protocol (PACMP) } \\
\text { Product Lifecycle Management } \\
\text { (PLCM) }\end{array}$ & $\begin{array}{c}\text { regulations } \\
\text { Location of PLCM document not } \\
\text { defined in regional regulations }\end{array}$ & $\begin{array}{c}\text { Location of PLCM document not defined in } \\
\text { regional regulations }\end{array}$ \\
\hline
\end{tabular}

this guideline the post approval change management system will be harmonized. There are still a number of issues to be addressed before the guideline can be fully implemented. But it is a difficult task to make changes in the existing regional regulations without developing a completely new formal procedure. It might also be difficult to harmonize the post approval process if the tools described in the guideline are not sufficiently defined in detail as there might be a scope for different interpretations of the guideline in different regions which might result in different requirements for the review authority of different regions. Moreover the maintenance of all the tools mentioned in the guideline would be quite complex and burdensome when practically implemented. The compatibility of the tools proposed in the guidelines with the regional regulations are very important. This might not only be challenging but it is of high importance that the implementation of the guideline be worthwhile.

The present review provided a detailed analysis of the current post approval regulations and process in India and Japan. Indian guidelines for post approval changes are not much well established, it's restricted only to biologics, risk based categorization and process is not clearly defined for new chemical entities. The establishment of EC into the Indian system will require strides of development in the established regulations as there is no similar concept present in existing regulations. Moreover implementation and maintenance of change management protocols and life cycle management document requires a complete regulatory overhaul and extensive time and resources. Compared to Japan, India is way behind and requires development in existing regulations to fully implement the harmonized ICH Q12 guideline.

Japan on the other hand has detailed guidance for post approval application along with risk based categorization of changes and submission process. With the similar concept called legally approved matters matching the requirement of EC as explained in the ICH Q12 guideline, Japan is a step ahead for fully realizing the ICH Q12 harmonized guideline. However Japan has to fully stream line the process and format as described in the harmonized guideline. Moreover the implementation of PACMPs is one of the challenges ahead for Japan. Japan's PMDA being one of the founder members of the ICH was already being considering the implementation of PACMP. The summary of the level of implementation of the $\mathrm{ICH}$ Q12 tools in both the countries is given in Table 3.

The implementation of the ICH Q12 guideline could allow more changes to be included under the pharmaceutical management system without the requirement of regulatory scrutiny. However it is expected to bring a large change in how post approval changes are managed in the global level. The guideline is expected to finish step 3 expert's signoff in June 2019 and adoption of step 4 by second half of $2019^{[20]}$.

\section{Acknowledgements:}

The authors thank Prof. Krishnamurthy Bhat, Head of department, Pharmaceutical Quality Assurance, Manipal College of Pharmaceutical Sciences, for providing the necessary facilities, to carry out the research work.

\section{REFERENCES}

1. Lokesh MS, Gupta NV, Belagoankar BD. Comparative study of process of post approval change application submission and approval for marketing authorization variations in EU, US, India, Saudi Arabia and Singapore. Int J Drug Dev Res 2015;7(1):0975-9344.

2. Van Buskirk G, Asotra S, Balducci C, Basu P, DiDonato G, Dorantes A, et al. Best practices for the development, scaleup, and post-approval change control of IR and MR dosage forms in the current quality-by-design paradigm. AAPS PharmSciTech 2014;15(3):665-93.

3. Mounica NVN, Reddy SV, Anusha S, Evangeline L, Nagabhushanam MV, Nagarjunareddy D, et al. Scale up and postapproval changes (supac) guidance for industry: a regulatory note. Int J Drug Regulatory Affairs 2017;5(1):1319.

4. Kumar P, Yadav V, Kaushik D. Post-approval changes in 
pharmaceuticals: regulatory perspectives in Europe. Appl Clin Res 2015;2(2):60-8.

5. Guidance for industry [Internet]. Cdsco.nic.in. 2019 [cited 14 November 2018]. Available from: http://www.cdsco.nic.in/ writereaddata/CDSCO GuidanceForIndustry.pdf.

6. Kuribayashi R, Matsuhama M, Mikami K. Regulation of generic drugs in Japan: the current situation and future prospects. AAPS J 2015;17(5):1312-16.

7. CDSCO [Internet]. Cdscoonline.gov.in. 2018 [cited 16 November 2018]. Available from: https://cdscoonline.gov.in/ CDSCO/homepage.

8. Pharmaceutical administration and regulations in Japan [Internet]. Jpma.or.jp. 2019 [cited 12 Oct 2018]. Available from: http://www.jpma.or.jp/english/parj/pdf/2017.pdf.

9. Mission: ICH [Internet]. Ich.org. 2019 [cited 13 April 2019]. Available from: https://www.ich.org/about/mission.html.

10. Process of harmonisation: ICH [Internet]. Ich.org. 2019 [cited 13 April 2019]. Available from: https://www.ich.org/products/ process-of-harmonisation.html.

11. Prajapati V, Dureja $H$. Product lifecycle management in pharmaceuticals. J Med Market 2012;12(3):150-8.

12. George M, Joseph L, Sandal S, Joseph S. Product life cycle management in regulated market of Europe. Pharm Regulatory Affairs 2017;06(01):121.

13. Technical and regulatory considerations for pharmaceutical product lifecycle management Q12 [Internet]. Ich.org. 2019 [cited 25 December 2018]. Available from: https://www.ich. org/fileadmin/Public_Web_Site/ICH_Products/Guidelines/ Quality/Q12/Q12_Draft_Guideline_Step2_2017_1116.pdf.

14. Kumar VP, Gupta NV. A review on quality by design approach (QBD) for pharmaceuticals. Int J Drug Dev Res 2015;7:52-60.

15. Members \& observers: ICH [Internet]. Ich.org. 2019 [cited 13 April 2019]. Available from: https://www.ich.org/about/ members-observers.html.

16. Articles of association [Internet]. Ich.org. 2019 [cited 11 April 2019]. Available from: https:/www.ich.org/fileadmin/ Public_Web_Site/ABOUT_ICH/Articles_Procedures/ ArticlesOfAssociation_Approved_2018_0606_v2.pdf.

17. Master file system for drug substances [Internet]. Pmda.go.jp. 2018 [cited 14 November 2018]. Available from: https://www. pmda.go.jp/files/000153373.pdf.

18. English | Japan Pharmaceutical Manufacturers Association (JPMA) [Internet]. Jpma.or.jp. 2019 [cited 11 April 2019]. Available from: http://www.jpma.or.jp/english/.

19. Desai KG, Colandene JD, Nesta DP. Japan common technical document: bracketing strategies for the manufacturing process. Biopharm Int 2017;30(5):32-7.

20. ICH Q12 EWG work plan [Internet]. Ich.org. 2019 [cited 11 April 2019]. Available from: https:/www.ich.org/fileadmin/ Public_Web_Site/ICH_Products/Guidelines/Quality/Q12/ Q12_EWG_WorkPlan_2019_0225.pdf. 